\title{
Human poisoning in South Africa - the knowledge gap
}

\section{What is known?}

According to the Global Burden of Disease (GBD) study in 2019, unintentional human poisoning, for all ages, accounted for $0.14 \%$ of global deaths and self-harm accounted for $1.34 \%{ }^{[1]}$ Despite the low contribution of human poisoning to global mortality, the World Health Organization (WHO) ${ }^{[2]}$ has long considered poisoning, both unintentional and as a result of self-harm, to be a significant public health issue. In fact, 2012 WHO data estimated that 193460 people died worldwide from unintentional poisoning during that year. Of these deaths, $84 \%$ occurred in low- and middle-income countries (LMICs).

Furthermore, almost 800000 people die due to suicide each year, ${ }^{[3]}$ and chemicals, particularly pesticides, account for a significant number of these deaths. Suicide is a global phenomenon but the data show that LMICS bear the brunt, with $79 \%$ of suicides in 2016 being reported from these countries.

What is known about poisoning in South Africa (SA)? In 2019, the GBD reported that unintentional human poisoning caused $0.098 \%$ of all deaths in SA, and self-harm accounted for $1.51 \%$ of all deaths. These values were less than, and slightly more than, the global averages, respectively. ${ }^{[1]}$ Being an upper middle-income country, ${ }^{[4]}$ these data are somewhat surprising and raise questions about the accuracy of overall poisoning data collection in SA. This notion is supported by a recent systematic review of the global distribution of acute unintentional pesticide poisoning by Boedeker et al. ${ }^{\left[{ }^{[5]}\right.}$ where SA was listed as one of only seven countries worldwide with limited data for their systematic analysis.

\section{What is new?}

While the paucity of data on human poisoning in SA has been acknowledged for some time, the study by Goga et al ${ }^{[6]}$ in this issue of the SAJCC helps to narrow this knowledge gap. Goga et al. ${ }^{[6]}$ describe the characteristics and experience of managing critically ill poisoned patients in a tertiary hospital intensive care unit (ICU), adding useful mortality predictors to the growing knowledge base for managing such patients in resource-constrained settings. It is worth noting that the reported ICU poisoning mortality rate of $16.5 \%$ was significantly higher than that reported in high-income countries $(2-3 \%)$.

Goga et al. ${ }^{[6]}$ reassuringly describe the mortality outcomes between patients referred from different levels of care in SA as being similar. This emphasises the importance of supporting healthcare workers at district levels of care to be able to assess, stabilise, and refer critically ill patients safely.

The overall spread of toxins causing severe poisoning described by Goga et al.$^{[6]}$ is similar to the data reported to the Poisons Information Helpline of the Western Cape, where $\sim 50 \%$ of moderate to severe poisonings are due to pharmaceuticals, $15 \%$ due to pesticides and $12 \%$ due to household products (C Stephen, unpublished data). The range of medications reported was also very similar apart from the lower incidence of calcium-channel blocker (CCB) poisonings described in Goga's study population. CCB poisoning may cause critical illness requiring intensive care resources. ${ }^{[7]}$

\section{What can be done?}

The poisons information services in SA have been growing steadily since the 1970s and have developed some valuable resources to support healthcare workers and the public. There are three Poisons Information Centres (PICs) in the state health sector, and the two PICs in Cape Town combined their expertise in 2015 to provide a 24-hour telephonic emergency helpline, the Poisons Information Helpline of the Western Cape ${ }^{[8]}$ to assist healthcare workers and members of the public in the assessment and management of acute poisonings. This service is underpinned by the poisons information database, AfriTox, ${ }^{[9]}$ developed and maintained by the PIC at Red Cross War Memorial Children's Hospital over the past 50 years. Both these resources are underutilised outside of the main metropolitan provinces of KwaZuluNatal, Gauteng and the Western Cape, yet they could contribute, not only to encouraging best practices in the assessment and management of acute poisoning, but also to the collection of acute poisoning data.

Apart from ensuring the optimal medical management of poisoned patients at all levels of care, medical practitioners may need reminders about the importance of reporting notifiable medical conditions (NMCs). ${ }^{[10]}$ This has been highlighted during the current COVID19 pandemic, where all probable or confirmed cases of COVID19 must be reported to the National Institute for Communicable Diseases (NICD). Pesticide poisoning, in all circumstances, including intentional, accidental or occupational, as well as lead and mercury poisoning, are all category two NMCs requiring reporting to the NICD within 7 days of the incident.

Pharmacovigilance, including an emphasis on effective child-proof packaging, as well as rational and careful prescribing by medical practitioners, would contribute to the reduction in availability of medications often used in self-harm poisoning, as well as in the accidental and often serious medication poisonings seen in children.

The strengthening of mental health services, with effective and evidence-based interventions at population, community and individual levels, should also be undertaken in an effort to prevent suicide and self-harming behaviour and reduce the critical care burden associated with these patients

Resources for critical care in SA are constrained, as is the case in many LMICs. While it is true that acute poisoning may lead to ICU admissions that are potentially preventable, utilisation of the available poisons resources in SA could contribute to streamlining the rational use of these limited critical care services, ensuring more comprehensive data collection, and ultimately improving patient outcomes.

\author{
C Stephen, MB ChB (UCT), DCH (SA) \\ Poisons Information Centre, Red Cross War Memorial Children's \\ Hospital and Department of Paediatrics and Child Health, Faculty of \\ Health Sciences, University of Cape Town, South Africa \\ cindy.stephen@uct.ac.za
}

South Afr J Crit Care 2021;37(1):4-5. https://doi.org/10.7196/SAJCC.2021.v37i1.493

1. Institute for Health Metrics and Evaluation (IHME). GBD Compare. Seattle: IHME, 2015. https://vizhub.healthdata.org/gbd-compare/ (accessed 5 March 2021). 
2. World Health Organization (WHO). Environmental Health in Emergencies. Geneva: WHO, 2021. https://www.who.int/environmental_health_emergencies/poisoning/en/ (accessed 5 March 2021)

3. World Health Organization. Mental Health and Substance Use. Geneva: WHO, 2019. https:// www.who.int/teams/mental-health-and-substance-use/suicide-data (accessed 5 March 2021).

4. The World Bank. World Bank Country and Lending Groups. Washington: The World Bank, 2021. https://datahelpdesk.worldbank.org/knowledgebase/articles/906519-world-bank-countryand-lending-groups (accessed 5 March 2021).

5. Boedeker W, Watts M, Clausing P, et al. The global distribution of acute unintentional pesticide poisoning: Estimations based on a systematic review. BMC Public Health 2020;20:1875. https:// doi.org/10.1186/s12889-020-09939-0
6. Goga R, de Vasconellos K, Singh D. Acute poisonings presenting to King Edward VIII Hospital intensive care unit. South Afr J Crit Care 2021:37(1):10-15.

7. Engebretsen K, Kaczmarek K, Morgan J, et al. High-dose insulin therapy in beta-blocker and calcium channel-blocker poisoning. Clin Toxicol 2011;49:277-283. https://doi.org/10.3109/15563 650.2011 .582471

8. Western Cape Government. Poisons Information Helpline of the Western Cape. https://www westerncape.gov.za/service/poisons-information-helpline-western-cape (accessed 5 March 2021).

9. AfriTox. https://www.afritox.co.za/ (accessed 5 March 2021)

10. National Institute for Communicable Diseases of South Africa. Notifiable Medical Conditions. Johannesburg: NICD, 2020. https://www.nicd.ac.za/nmc-overview/ (accessed 5 March 2021). 